\title{
Correction to: Fetal MRI
}

\author{
Keiko Segawa
}

\section{Correction to:}

Chapter 3 in: H. Masuzaki (ed.), Fetal Morph Functional

Diagnosis, Comprehensive Gynecology and Obstetrics, https://doi.org/10.1007/978-981-15-8171-7_3

We inadvertently published the figures " $3.1,3.3,3.4,3.5,3.6$ " without the arrow marks. This has been rectified in this version. 

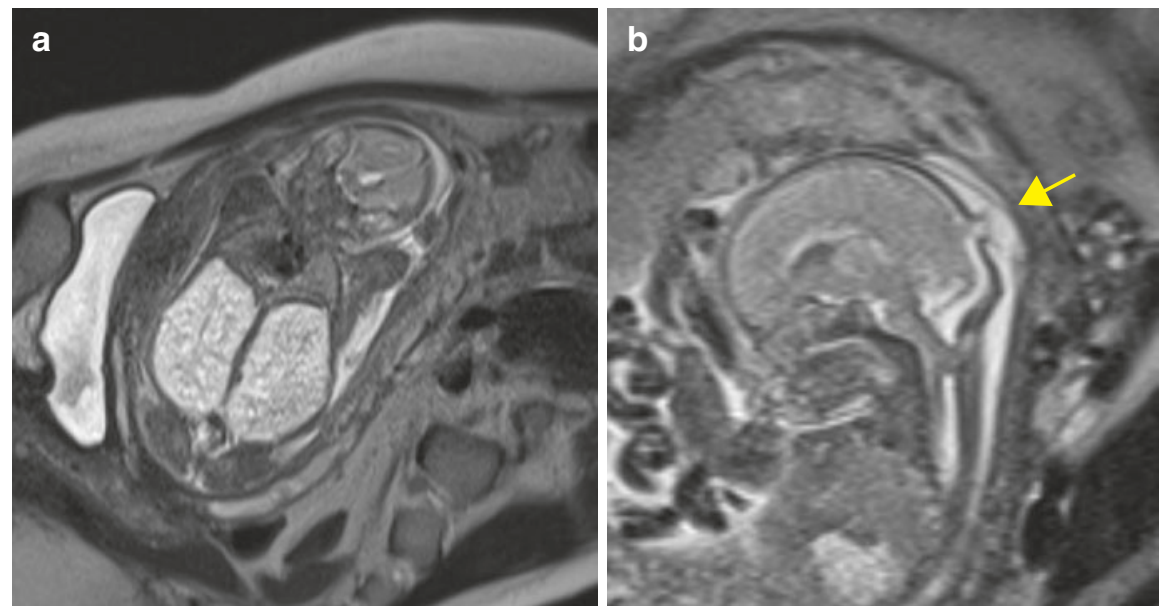

Fig. 3.1 (a) Coronal MRI of the fetus at 20 weeks shows severe oligohydramnios. The kidneys are grossly enlarged with abnormal hyperintense parenchyma. (b) Sagittal MRI of the brain in the same fetus shows an occipital calvarial defect and posterior encephalocele (arrow). These findings are classic features in Meckel-Gruber syndrome 

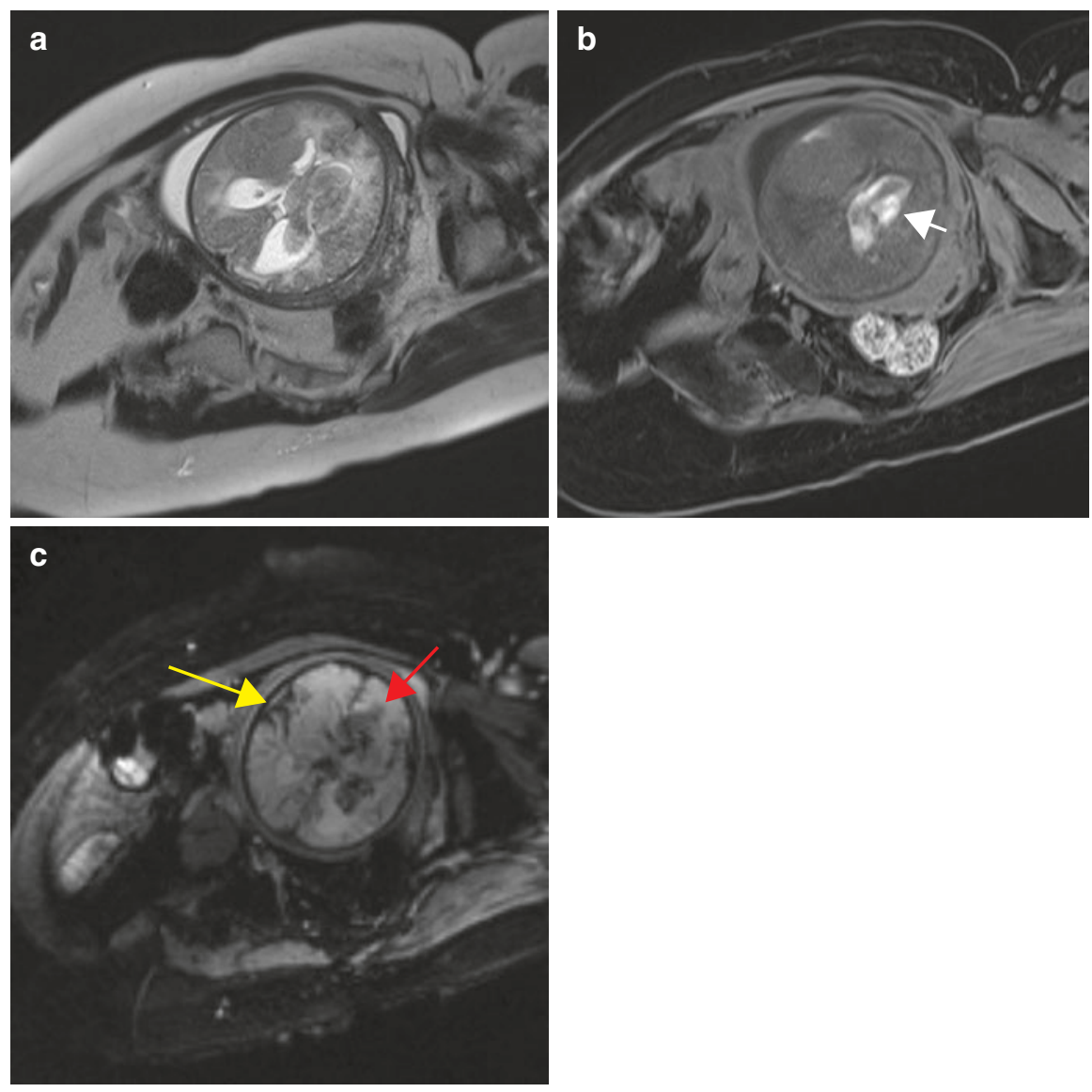

Fig. 3.3 Axial MRI of different sequences of the fetus at 37 weeks with ventriculomegaly and intraventricular hemorrhage. (a) T2-weighted image. (b) T1-weighted image. (c) Echoplanar imaging. Left caudate nucleus shows swelling with ventricular hematoma showing T2-hypointensity/ T1-hyperintensity (white arrow). On EP imaging, right Sylvian fissure subarachnoid hemorrhage (yellow arrow) and parenchymal hemorrhage in the left frontal lobe (red arrow) are clear 

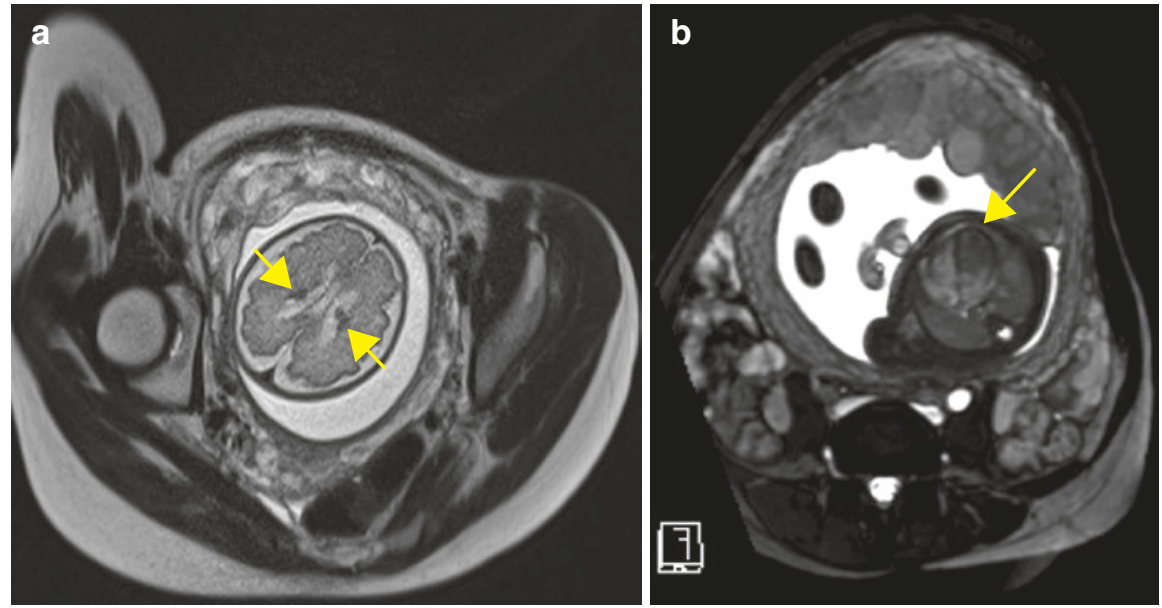

Fig. 3.4 Rhabdomyomas and tuberous sclerosis complex. MRI of the fetus at 29 weeks. (a) Axial T2-weighted image shows subependymal nodules (arrows). (b) SSFP sequences demonstrate cardiac rhabdomyomas as hypointense area (arrow)
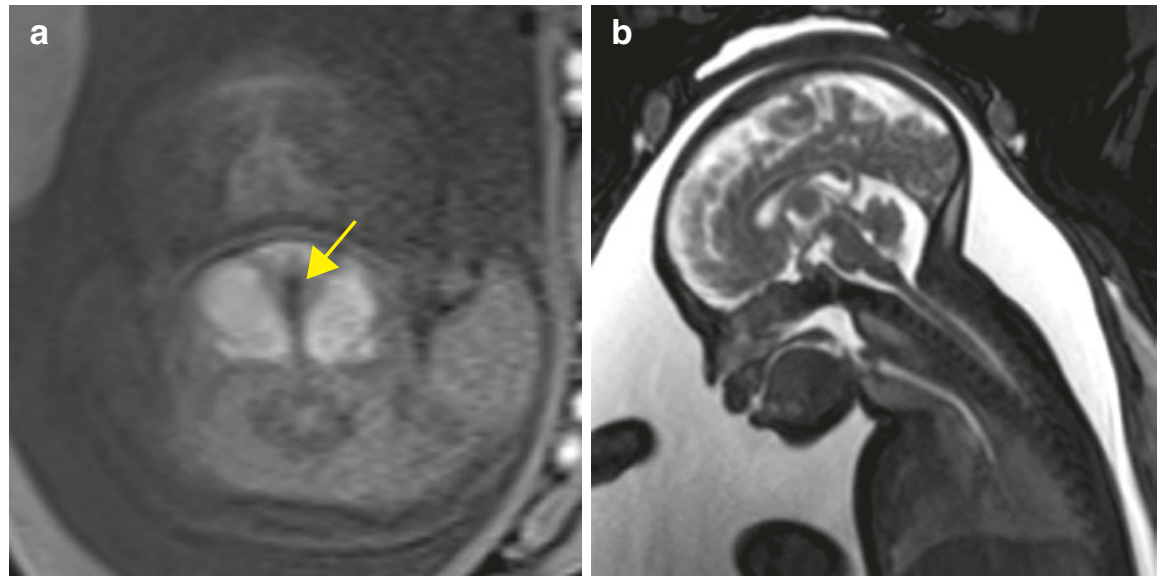

Fig. 3.5 MRI of the fetus at 35 weeks with large goiter. (a) On axial T1-weighted image, the large goiter showed hyperintensity and the trachea running in the center was narrowing (arrow). (b) Fetal airway patency was assessed with 3D dataset for reconstruction 

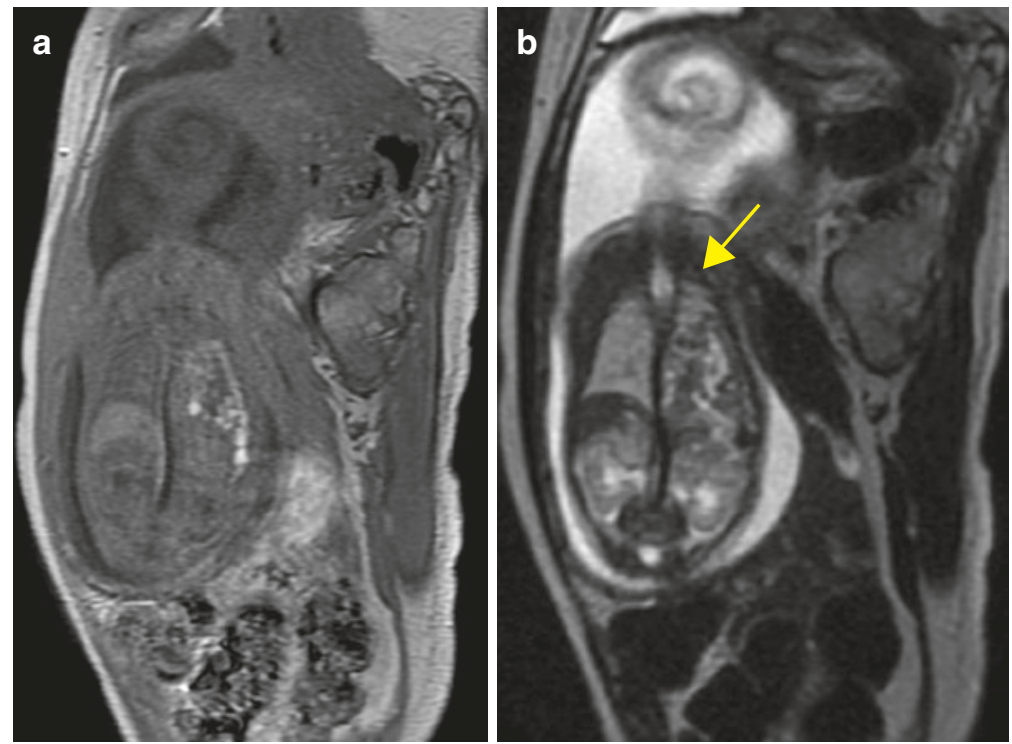

Fig. 3.6 Coronal MRI of the fetus at 28 weeks with congenital diaphragmatic hernia. (a) T1-weighted sequence reveal the presence of meconium-filled bowel within the thoracic cavity. (b) T2-weighted sequences reveal a tiny left lung at the top of thoracic cavity (arrow) 